\title{
Sinus Tract Formation with Chronic Inflammatory Cystic Mass after Beta Tricalcium Phosphate Insertion
}

\author{
Hong Jin Kim, \\ Woong Gyu Na, \\ Sung Won Jung, \\ Sung Hoon Koh, \\ Hyoseob Lim
}

Department of Plastic and Reconstructive

Surgery, Hallym University Sacred Heart

Hospital, Hallym University, Anyang, Korea

\begin{abstract}
Beta tricalcium phosphate ( $\beta$-TCP) is one of allogenic bone substitute which is known to have interconnected pores that draws cell and nutrients for bone generation. It has been resulted in good outcomes for bone defect coverage or augmentation. However, several studies have also reported negative outcomes and associated complications including unexpected formation of cystic mass, continuous pain and secretion. We present the case of a 36-year-old man with a right cheek cystic mass who had a history of right zygomaticomaxillary (ZM) complex fracture and surgical correction with $\beta$-TCP powder insertion to ZM bone defect. Excisional biopsy under local anesthesia revealed calcified mass in a sinus tract which was found to be connected to the ZM bone defect site in postoperative computed tomography image. Further excision under general anesthesia was performed to remove the sinus tract and fine granules which filled the original defect site. Pathologic report revealed bony spicules and calcification materials with chronic foreign body reaction. Postoperative complications and recurrence were not reported.
\end{abstract}

Keywords: Beta tricalcium phosphate / Complication / Foreign body reaction

\section{INTRODUCTION}

Allogenic bone substitutes that replace autogenous bone grafts, should be biocompatible, non-antigenic, and degradable with osteogenic capacity, and are constant pursuits in maxillofacial surgery $[1,2]$. Beta tricalcium phosphate $(\beta-T C P)$ is one such biomaterial. Although good outcomes have been reported for bone defect coverage or bone augmentation [2-5], several studies have also reported negative results when filling bone defects with $\beta$-TCP, including poor bone formation, unexpected formation of cystic mass, continuous pain, and secretions, which may attribute to soft tissue inflammation after insertion of $\beta$-TCP $[1,6-8]$. We present the case of a 36-year-old man with a right cheek cystic mass who

\section{Correspondence: Hyoseob Lim}

Department of Plastic and Reconstructive Surgery, Hallym University Sacred Heart Hospital, Hallym University, 22, Gwanpyeong-ro 170 beon-gil, Dongan-gu, Anyang 14068, Korea

E-mail: hyoseob.lim@gmail.com

Received Oct 12, 2017 / Revised Nov 6, 2017 / Accepted Nov 14, 2017 had a history of right zygomaticomaxillary (ZM) complex fracture and surgical correction with $\beta$-TCP powder insertion. The mass was initially believed to be an epidermal cyst, but histologically demonstrated calcified clusters and bony spicules, which may be due to the failure of $\beta$-TCP bone formation.

\section{CASE REPORT}

A 36-year-old man visited our department with a mass on his right cheek, measuring $1 \times 1 \mathrm{~cm}$ lateral to the oral commissure. He had undergone surgical correction for right ZM complex fracture with bone defect at ZM buttress and right inferior orbital wall fracture due to road traffic accident 6 years prior. The bone defect was filled with $\beta$-TCP bioceramic powder (PolyBone, Kyungwon Medical Co. Ltd., Seoul, Korea). The mass had appeared 5 years prior along with signs of inflammation of the adjacent skin, such as redness and tenderness (Fig. 1). Initially, excisional biopsy through direct skin incision under local anesthesia was per- 
formed with a preliminary diagnosis of infected epidermal cyst. However, the mass was calcified and had a sinus tract (Fig. 2). The tract was not completely removed as it extended into the deep tissue. Biopsy results revealed chronic inflammation around the calcified fragments. Computed tomography (CT) with contrast enhancement revealed that the sinus tract was connected to the $\mathrm{ZM}$ bone defect site (Fig. 3). Bone formation was not seen at the defect site and remnant calcified granules were found above the defect.

Further surgical resection under general anesthesia was performed to remove the sinus tract and remnant granules completely. Intraoral approach with right upper buccogingival incision was used to reveal the fibrous sinus tract connecting the defect to the subcutaneous tissue. The tract was excised from the superficial fat tissue, and resected in an en bloc pattern with the help of a guide probe (Fig. 4). Dense adhesive tissue at the defect was also completely removed and curettage of the fine granules filling the ZM defect was performed with copious saline irrigation. The fine granules were connected to skin through the sinus and covered by a cystic wall, which isolated it from the adjacent bony structures. The Stensen's duct was intact. Histopathological examination revealed that it was a foreign body reaction with chronic inflammation of the sinus tract and that the tiny granule was degenerated bony spicules and calcification materials (Fig. 5). The surgical wound healed without any complication and no recurrence was noted.

\section{DISCUSSION}

The maxillary bone is related to facial aesthesis and several oral functions such as speech, swallowing, and mastication. It supports the orbital structures, and separates the oral and orbital cavities [9]. Autogenous bone graft is considered the material of choice for the reconstruction of bone in maxillofacial surgery [1]. However, to prevent secondary donor site morbidity and increased duration of the operation for the harvesting procedures, several artificial bone substitutes, including bioceramics, allograft bone matrix, and composites have been developed for craniofacial defects [4].

The major inorganic component of bone tissue is hydroxyapa-

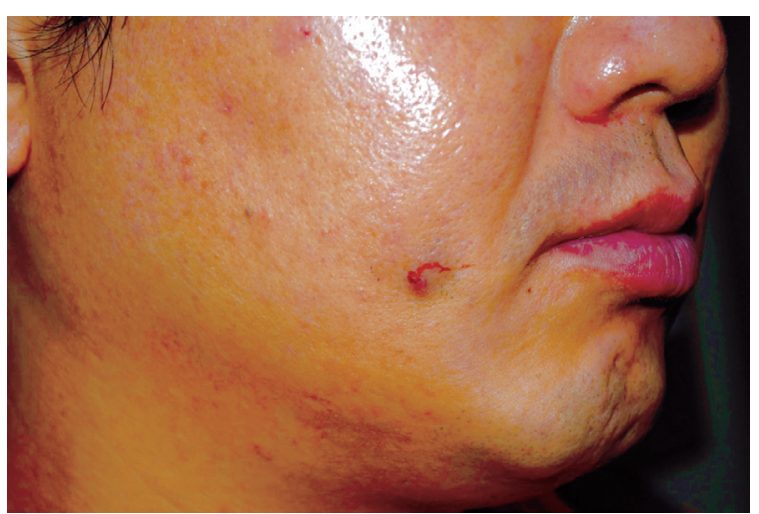

Fig. 1. The mass on the right cheek with inflammatory signs. Preoperative photography.
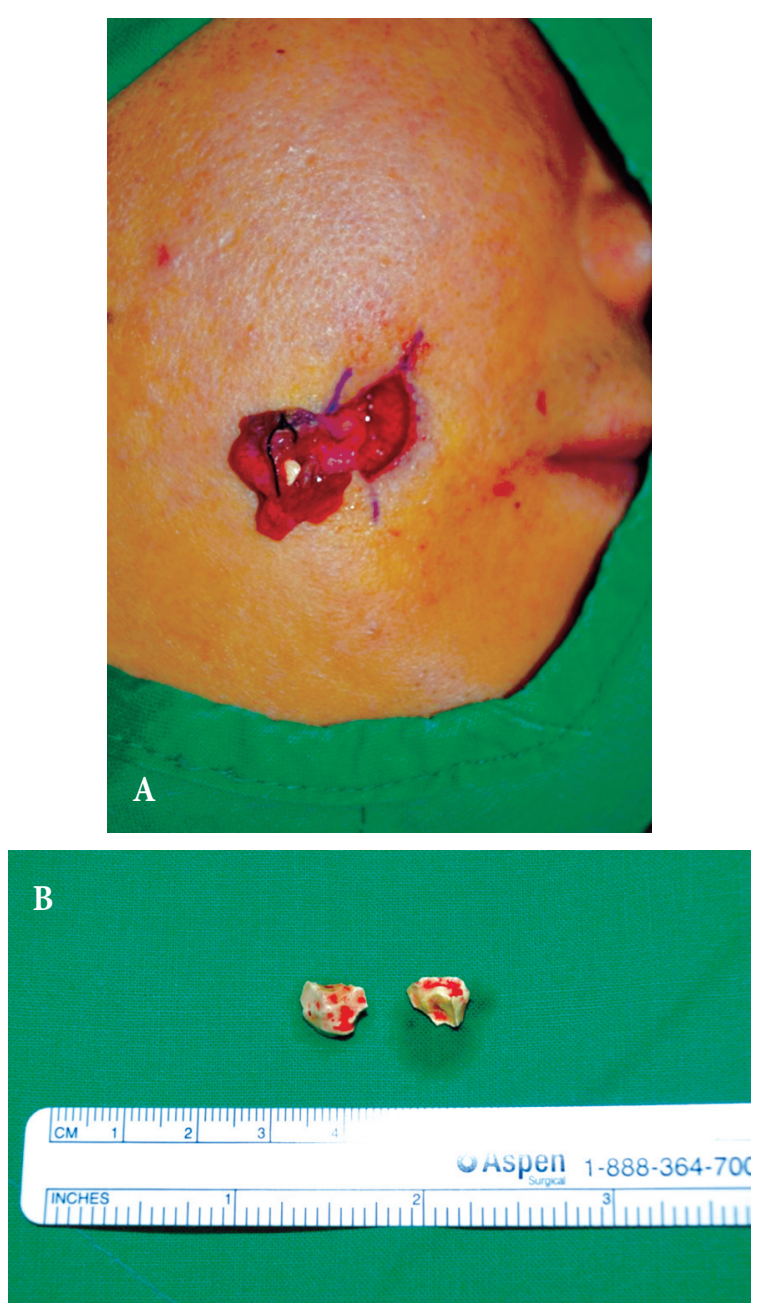

Fig. 2. (A) Excised sinus and calcified fragments at first operation. (B) Calcified masses removed. 

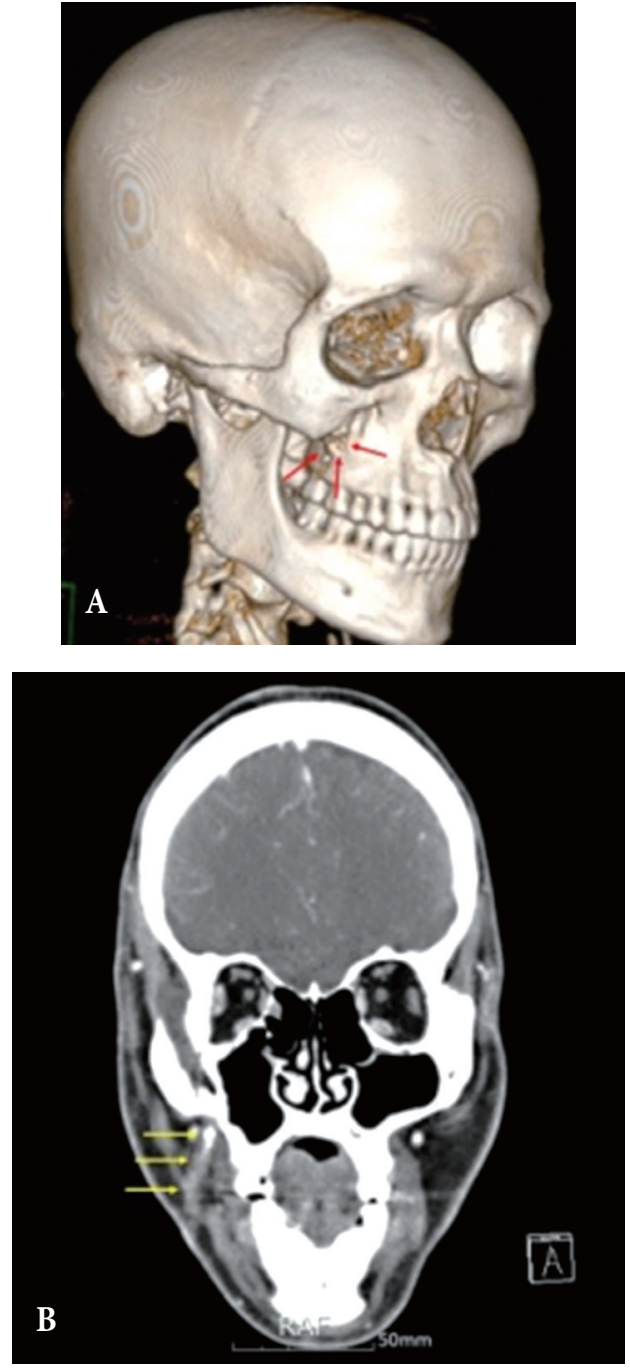

Fig. 3. (A) Zygomaticomaxillary buttress defect (red arrows) was shown. (B) Computed tomography with contrast enhancement revealed that the sinus tract (yellow arrows) was connected to the defect site.

tite (HA), and many different calcium phosphate ceramics have been utilized as a material for bone substitutes. As a bone substitute, HA is highly osteocompatible and expresses sufficient osteoconductivity, but stoichiometric HA is unbioresorbable, and has much less mechanical strength than bone tissue because there is no organic component such as type I collagen in the bone substitute. Therefore, the HA that remains in bone tissue can cause serious clinical problems [10].

$\beta$-TCP is a bioceramic material and is known to have interconnected pores that create a capillary force to draw cells and nutri-
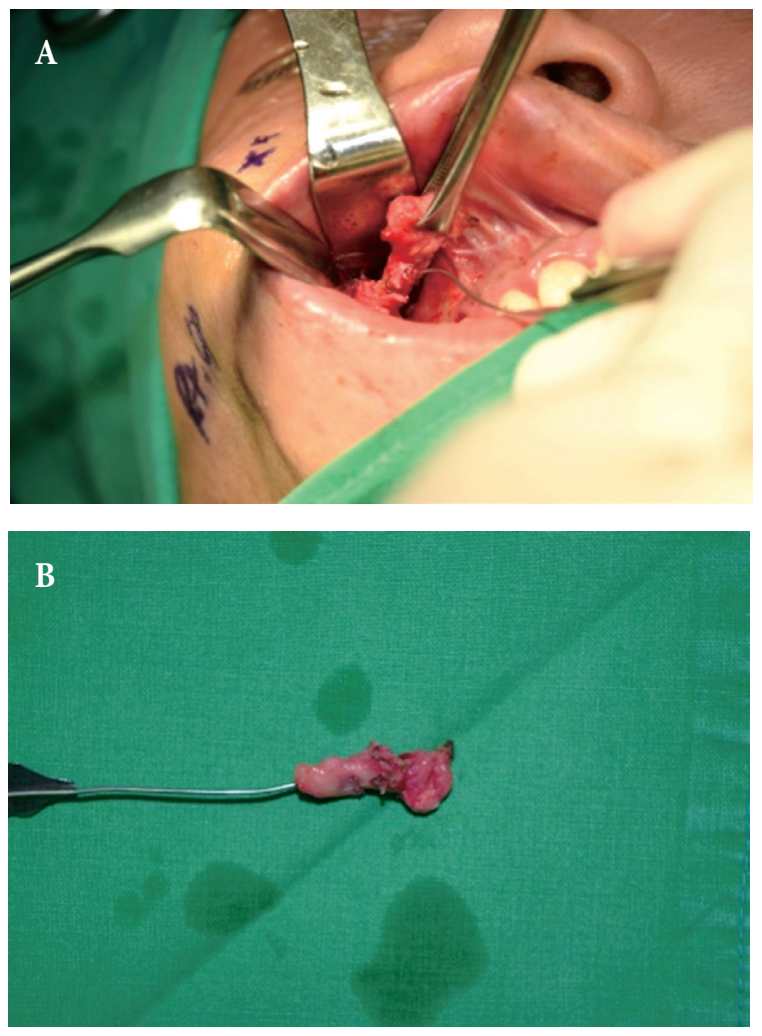

Fig. 4. (A) Buccogingival approach revealing fibrous sinus. (B) Excised sinus with probe guide at second operation.

ents into the material for bone generation [2]. It resolves completely in the human body in a centripetal pattern followed by new bone formation $[4,6]$, so has used as most popular bioresorbable materials for bone substitutes. $\beta$-TCP, alone or mixed with other bioceramics, has been reported to have favorable outcomes in maxillary sinus augmentation for dental implant fixation and reconstruction of the orbital floor and bone defects followed by bone tumor resection and curettage [2-5].

Adverse effects of $\beta$-TCP have also been reported. Poor osteogenic effect has been observed in several in vivo studies $[2,6,8]$. Friesenbichler showed that bone defects covered with $\beta$-TCP mixed with calcium sulfate could result in soft tissue inflammation leading to delayed wound healing, continuous wound secretion and pain, and formation of cysts [7]. In this case, follow up CT revealed bone defect in the right ZM buttress area implying failure of bone formation and that calcified fragments and bony spicules were located away from fracture site, in a sinus connected 

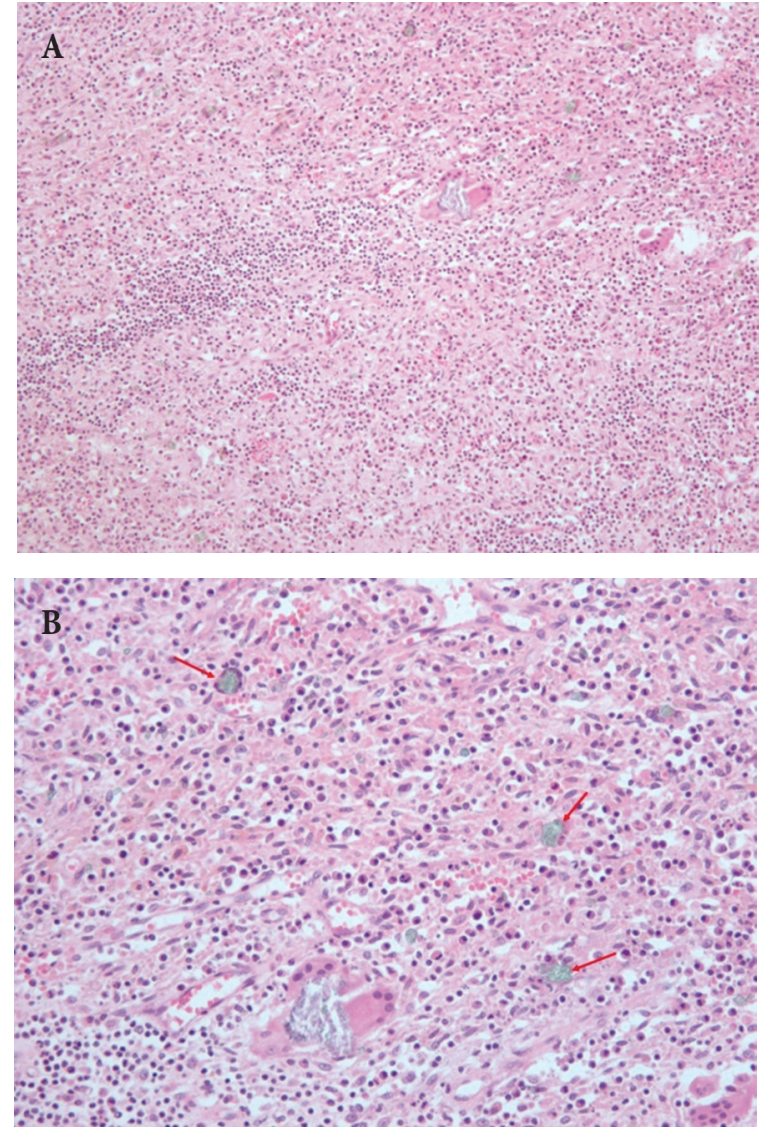

Fig. 5. (A) Multiple infiltrated lymphocytes were shown (H\&E, $\times 100$ ). (B) Some calcified materials (red arrows) were confirmed that causing foreign body reaction $(\mathrm{H} \& \mathrm{E}, \times 200)$.

to the defect site. Histologically, chronic foreign body reaction was found even 6 years after insertion of $\beta$-TCP. Foreign body reaction is a response of wound healing following the insertion of biomaterials and it may play a major role in the formation of bony spicules, calcified clusters, and sinuses $[1,10,11]$. de Souza et al. reported foreign body reaction and inflammatory infiltrates in $\beta$-TCP insertion sites; they hypothesized that it may lead to expulsion of $\beta$-TCP clusters from the original defect site [1]. Sinus formation resulting from foreign body reaction was also reported [12].

In conclusion, because of the debatable findings regarding $\beta$ -TCP demonstrated in our case and several other studies, informed consent is very important when filling maxillofacial defects with $\beta$-TCP about the possibility of failure of bone formation along with formation of cysts and inflammatory complications, including pain, delayed wound healing, continuous discharge, and sinus formation. Additionally, long-term follow up with radiographic examination including X-ray and CT is needed for evaluating bone formation.

\section{CONFLICT OF INTEREST}

No potential conflict of interest relevant to this article was reported.

\section{PATIENT CONSENT}

The patient provided written informed consent for the publication and the use of their images.

\section{REFERENCES}

1. de Souza DF, Correa L, Sendyk DI, Burim RA, da Graca NaclerioHomem M, Deboni MC. Adverse effect of beta-tricalcium phosphate with zeta potential control in repairing critical defects in rats' calvaria. Rev Bras Ortop 2016;51:346-52.

2. Pereira RS, Gorla LF, Boos F, Okamoto R, Garcia Junior IR, HochuliVieira E. Use of autogenous bone and beta-tricalcium phosphate in maxillary sinus lifting: histomorphometric study and immunohistochemical assessment of RUNX2 and VEGF. Int J Oral Maxillofac Surg 2017;46:503-10.

3. Annibali S, Iezzi G, Sfasciotti GL, Cristalli MP, Vozza I, Mangano C, et al. Histological and histomorphometric human results of HA-BetaTCP 30/70 compared to three different biomaterials in maxillary sinus augmentation at 6 months: a preliminary report. Biomed Res Int 2015;2015:156850.

4. Bittermann GK, Janssen NG, van Leeuwen M, van Es RJ. One-year volume stability of human facial defects filled with a beta-tricalcium phosphate-hydroxyl apatite mixture (Atlantik). J Craniofac Surg 2014;25:372-4.

5. Seto S, Muramatsu K, Hashimoto T, Tominaga Y, Taguchi T. A new beta-tricalcium phosphate with uniform triple superporous structure as a filling material after curettage of bone tumor. Anticancer Res 2013;33:5075-81.

6. Auston DA, Feibert M, Craig T, Damron TA. Unexpected radiographic lucency following grafting of bone defects with calcium sulfate/tricalcium phosphate bone substitute. Skeletal Radiol 2015;44: 1453-9.

7. Friesenbichler J, Maurer-Ertl W, Sadoghi P, Pirker-Fruehauf U, Bodo $\mathrm{K}$, Leithner A. Adverse reactions of artificial bone graft substitutes: lessons learned from using tricalcium phosphate geneX(R). Clin Orthop Relat Res 2014;472:976-82. 
8. Orii H, Sotome S, Chen J, Wang J, Shinomiya K. Beta-tricalcium phosphate (beta-TCP) graft combined with bone marrow stromal cells (MSCs) for posterolateral spine fusion. J Med Dent Sci 2005;52:51-7.

9. Iyer S, Thankappan K. Maxillary reconstruction: Current concepts and controversies. Indian J Plast Surg 2014;47:8-19.

10. Okuda T, Ioku K, Yonezawa I, Minagi H, Kawachi G, Gonda Y, et al. The effect of the microstructure of beta-tricalcium phosphate on the metabolism of subsequently formed bone tissue. Biomaterials 2007;28:2612-21.

11. Anderson JM, Rodriguez A, Chang DT. Foreign body reaction to biomaterials. Semin Immunol 2008;20:86-100.

12. Hansen KB, Gottrup F. Chronic ulceration and sinus formation due to foreign body: an often-forgotten problem. Int J Low Extrem Wounds 2015;14:393-5. 